\title{
Bioengineering of nano drug delivery compound to enhance the bioavailability of selected bioactive compound
}

\section{Introduction}

Bioengineering refers to the application of various disciplines of Engineering and its principles to design, develop and analysis to biological systems (Both plant and animals). This is a rapidly growing field of engineering which is the integration of engineering, biology, pharmaceuticals, medicines, agriculture, natural resources and food stuffs. Drug delivery systems are engineered technologies for the targeted delivery and/or controlled release of therapeutic agents.

There are phyto chemicals, which are either poorly soluble or completely fat soluble. It is known that the delivery of these phyto chemicals is significantly influenced by their physicochemical properties, such as water solubility, partition coefficient, lipophilicity and crystallinity, and so on. It is been reported that, the oral bio availability of various phyto constituents range from $13-19 \%$. To have higher bio availability, it is better to be formulated into a highly bio available compound using bio availability enhancers. A major obstacle in the development of phytochemicals-based drug has been the lower water solubility which makes it difficult to develop effective formulation for human use. This Nano emulsion based delivery system have been proved to be one of the best platforms to enhance the bio availability and biological effectiveness of phyto chemicals.

The nano emulsions based drug delivery systems must have the following advantages (i) high kinetic or thermodynamic stability, which provides significantly better stability over unstable dispersions. (ii) either hydrophilic or lipophilic phytochemicals can be incorporated into the same nanoemulsions; and (iii) because of the small droplet sizes $(65-75 \mathrm{~nm}, \mathrm{r})$, phyto chemicals can be transported through the cell membranes much more easily, resulting in an increased phyto chemicals concentration in plasma and bioavailability.

Nano emulsions are referred as, the emulsions that has the droplet size range of $20-500 \mathrm{~nm}$. Nano emulsions, unlike conventional emulsions or micro emulsions, are having several differences in their physicochemical properties such as long term stability, high bioavailability etc. Nano emulsions are thermodynamically stable systems used to deliver highly lipophilic drugs. The oral bioavailability of highly lipophilic drug molecules can be increased by self emulsifying nano emulsion drug delivery system.

These nano emulsions can be engineered using high energy methods and low energy methods.

a. High energy methods: Mechanical devices.

b. Low energy methods: Self emulsification (SE)/Spontaneous emulsification.

Self emulsifying formulations (SEF) are mixture of oil, surfactant, co surfactant, co -solvent, which forms a transparent solution, which emulsify under gentle mixing, similar to that happens in the GI tract. These SEF, when orally administered undergo spontaneous emulsification along with GI fluids. ${ }^{1-5}$

\author{
Volume 8 Issue 3 - 202I
}

\author{
S Prem Mathi Maran \\ Research Director and Principal Scientist, Clean Green Bio \\ Research Foundation, India
}

Correspondence: S Prem Mathi Maran, Research Director and Principal Scientist, Clean Green Bio Research Foundation, Plot No 160, Karpagam Nagar, Thirumudivakkam, Chennai 600044, India, Email drpremmathimaran@gmail.com

Received: April 23, 2021 | Published: June 28, 2021

\section{Bioavailability enhancing property of nano emulsions}

The bio availability enhancing property of self emulsifying formulations are attributed to a number of in vivo properties, which includes:

a) The inhibition of cellular efflux mechanisms.

b) Reduction of first pass drug metabolism in the liver due to association of certain lipid excipients with selective drug uptake into the lymphatic circulation system.

c) The formation of fine dispersions and miscella suspensions to prevent precipitation and re crystallization of drug compounds.

d) The ability of certain lipid compounds and their metabolites to initiate changes in the GI fluid, in favour of improved drug absorption. ${ }^{6-15}$

\section{Bioenhancers}

Bioenhancers are defined as substances that increase the bioavailability and bioefficacy of active substances with which they are combined without having any activity of their own at the dose used. Besides several classes of modern drugs like antibiotics, anti cancer drugs, cardiovascular drugs, anti inflammatory, central nervous drugs, etc., they also increase the bioavailability of vitamins and nutrients. Increased Bioavailability means increased levels of drug in the blood stream available for drug action. Increased Bioefficacy means the increased effectiveness of the drug due to increased bioavailability and also due to other mechanisms. 


\section{Bioavailability}

Bioavailability is a measure of the amount of an administered dose that reaches the bloodstream. Thus, it represents the fraction of the dose that is absorbed and escapes first-pass elimination.

Bioavailability is typically estimated by measuring a portion of a drug (as a percentage) which reaches the systemic blood flow after its non-systemic administration.

Bioavailability, which is the key pharmacokinetic property of any phyto pharmaceuticals drug. Phytochemicals such as polyphenols (curcumin, resveratrol, catechins), carotenoids (lycopene, beta carotene etc) having various health benefits. These phytoconstituents receive much attention from scientific communities, phytopharma and nutraceuticals industry, food manufacturers as well as consumers.

Though, the use of tea polyphenols as capsules and tablets are abundant, their biological activity is frequently diminishing or even lost due to incomplete metabolism and first pass metabolism. A review by Manach. on the oral bioavailability of 97 polyphenols showed that, proanthocyanidins, galloylated tea catechins, curcumin and anthocyanins are least absorbed polyphenols.

Until recently the research focus on solubility of lycopenes, phytosterols and few other phytoconstituents. The water soluble nano emulsion of green tea catechins are still limited. Hence, we undertook a research to produce a highly bioavailable Gallate catechins extract by a new methodology of using fresh green tea leaves and formulating the green tea extract into a water soluble nano emulsion.

There are various methods and techniques are there to enhance the bioavailability of phytochemicals among those Nano emulsions is one of the efficient techniques to enhance the bioavailability of the drugs, these are nano-scaled emulsions, which are generally prepared by various methods. Here in the method employed to produce nano emulsion was high shear-high energy homogenization method. ${ }^{10}$ Nano and have greater surface area providing greater absorption. As a drug delivery system, the Nanoemulsion enhance physical stability and bioavailability of drug, and therefore the therapeutic efficacy of drug can be improved, minimizing adverse effect and toxic reactions.

\section{Materials and methods}

\section{Reagents and chemicals}

Cremophor-EL was procured from BASF, Germany. Ethanol (purity 99.8\%) was procured from Merck. Poly Ethylene Glycol (PEG)-Purity 99.9\% was obtained from Sigma-Aldrich. Glycerin (99.9\% purity) was procured from pharmacy (Figure 1).

\section{Plasma concentration (ng/ml of plasma) of total catechins of reference green tea extract, hydyne processed extract and nano formulation}

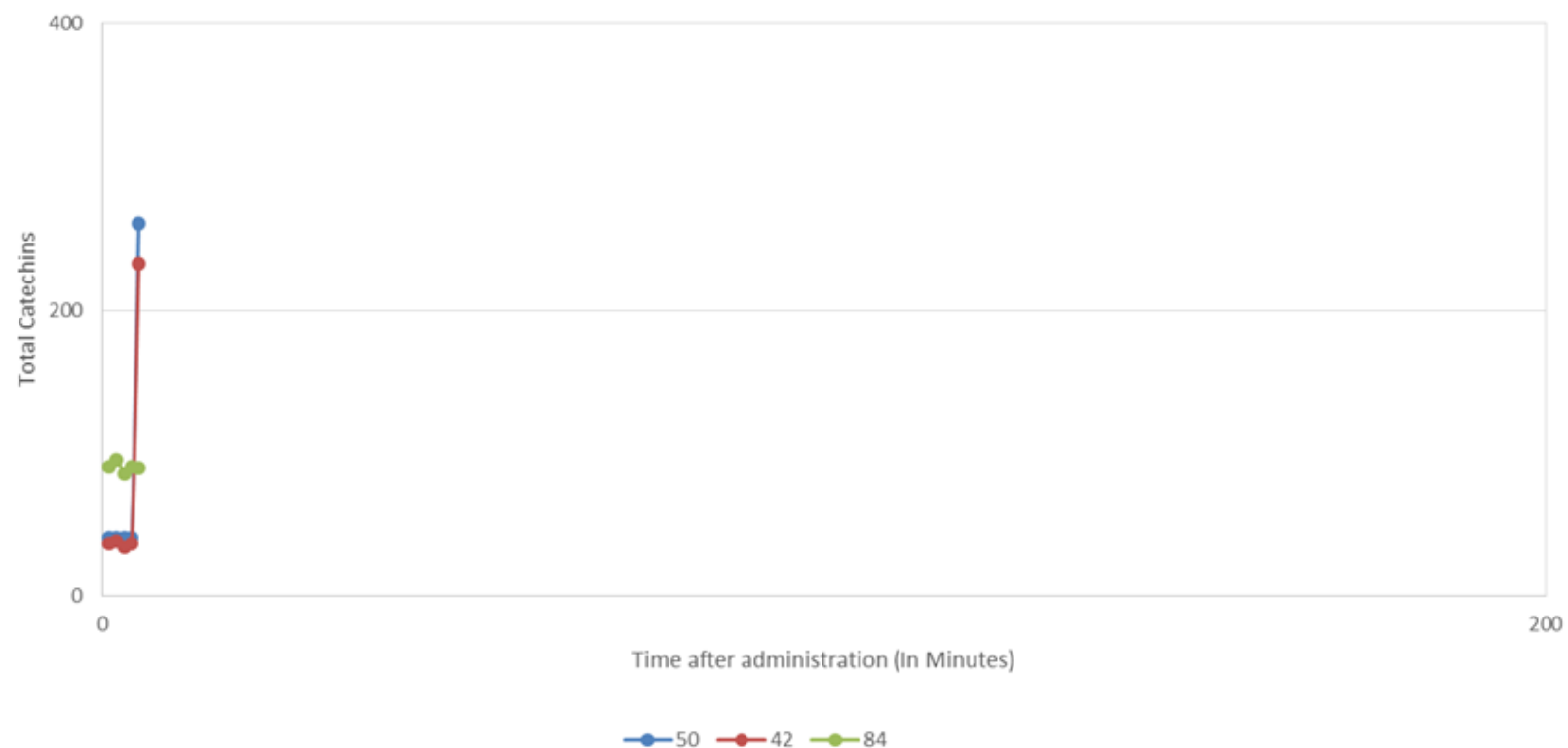

Figure I Plasma concentration ( $\mathrm{ng} / \mathrm{ml}$ of plasma) of total catechins of reference green tea extract, hydyne processed extract and nano formulation.

\section{Extraction of botanical biopotentiators}

Equal amount of black pepper (Piper nigrum), zinger (Zingiber officinale) and long pepper (Piper longum) were taken, mixed and size reduced to a coarse powder to a bulk density of 0.4 . Using a soxhelet extractor and using grain alcohol as a solvent, the extraction of combined biopotentiators was done for 4 hours. After 4 hours, the miscella was collected and the solvent was distilled using a rotary evaporator. A thick green coloured paste was obtained, which was used as a botanical biopotentiators in the nano emulsion formulations.

\section{Nano emulsion preparation}

Nano emulsion was prepared using spontaneous emulsification method as prescribed by Bouchemal with a modification. (Flow chart no....). The reactor speed (rpm), reaction time, reaction temperatures and dilution procedures, order of addition of various ingredients are carefully engineered in order to ensure a uniform droplet size of 65 to $75 \mathrm{r}, \mathrm{nm}$ and uniform droplet size, uniform distribution and stability of the nano emulsion (Figure 2). 


\section{BOTANICAL BIOPOTENTIATORS}

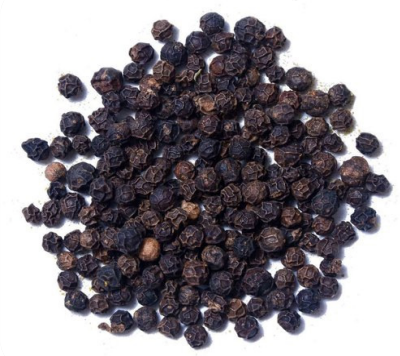

BLACK PEPPER
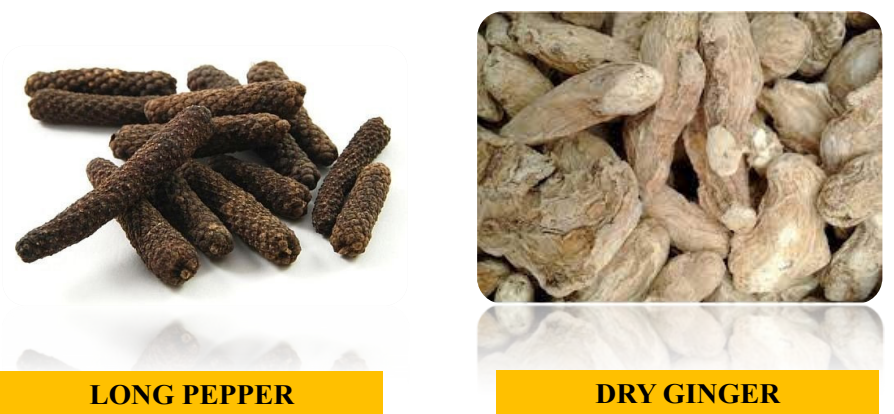

\section{BIO ENGINEERING THE NANO EMULSION}

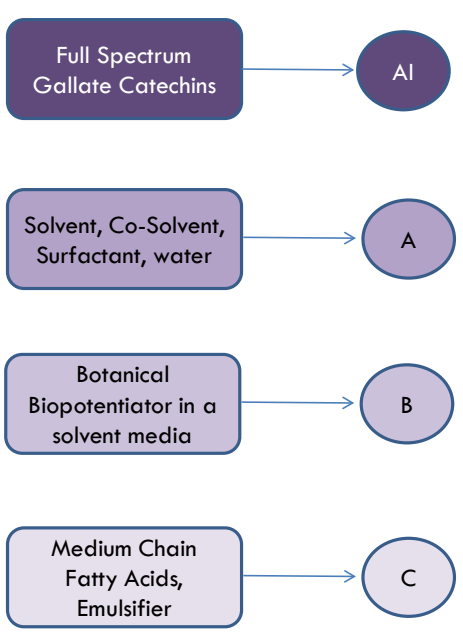

Figure 2 Bioengineering the Nano emulsion.

\section{Droplet size and poly dispersity study}

The droplet size measurement was done using a Dynamic Light Scattering Methods (DLS). At $633 \mathrm{~nm}$ laser wavelength and $173 \mathrm{Deg}$ angle, the z-average droplet size, particle size distribution (PSD) and poly dispersity index (PDI) were studied.

\section{Plasma concentration study}

Group of healthy volunteers were enrolled in the study. Before the start of the study participants were physically examined and a blood sample was taken to evaluate the health condition of the participant During this check-up the following demographic and diagnostic parameters were collected: gender, age, weight, height, BMI, typical clinical biochemistry parameters (AST, ALT, Bilirubin), complete blood count, green tea consumption habits, smoking status, frequency of alcohol consumption and use of oral contraceptives.
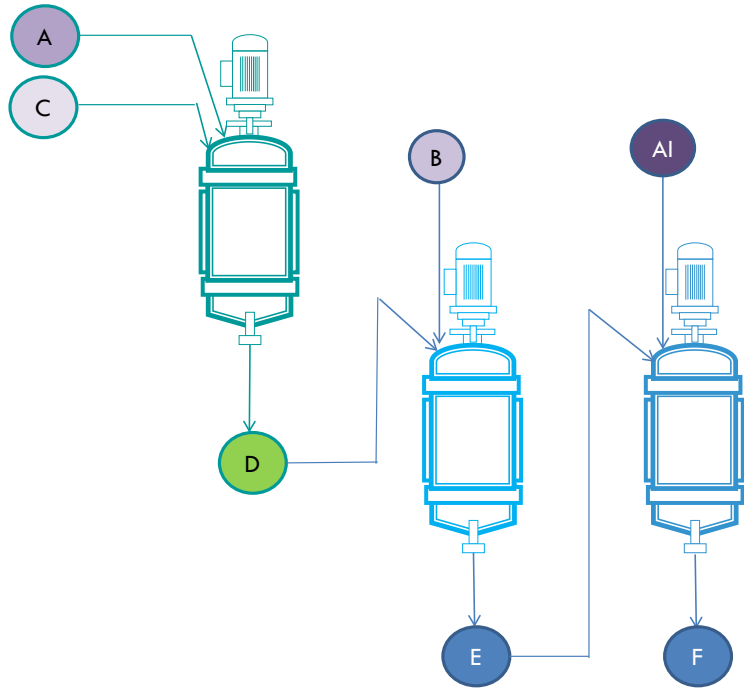

\section{Study design}

One day before the treatment period participants were requested to stop any green tea consumption and to avoid polyphenol rich nutrition during study. All participants were asked if they did extensive physical exercises in the days before and during the study. None of the participants stated an extensive physical activity. For the first 4 days of the treatment period participants took green tea capsules twice daily (气 $2 \times 150 \mathrm{mg} /$ day). Capsules were swallowed in the morning and in the evening ( $12 \mathrm{~h}$ interval) with a glass of tap water during meal. After one-night fasting, on the morning of day 5 a blood sample for the baseline measurements was taken. Afterwards another capsule of green tea extract (150 mg EGCG) was administered and subsequently venous blood samples were taken after . A standardized meal and snack were served $4 \mathrm{~h}, 8 \mathrm{~h}, 10 \mathrm{~h}, 12 \mathrm{~h}$ and $24 \mathrm{~h}$ after the intake of the green tea extract capsule. 


\section{Tea polyphenol concentration measurement}

Venous blood was drawn into ethylene diamine tetra acetic acid (EDTA) containing tubes and centrifuged within 10 minutes after sampling at $3500 \mathrm{rpm}$ for 10 minutes. Then, $1 \mathrm{~mL}$ of plasma were mixed with $20 \mu \mathrm{L}$ of antioxidant solution $(2 \mathrm{~g}$ ascorbic acid per $10 \mathrm{~mL}$ double distilled water including $70 \mu \mathrm{L}$ of $0.5 \mathrm{M}$ EDTA) and stored at $-80^{\circ} \mathrm{C}$ until analysis.

Calibrator and IS (Internal standard) stock solutions were prepared as $1 \mathrm{mg} / \mathrm{mL}$ solutions in $50 \%$ methanol including $2 \%$ of the antioxidant solution given above. Calibrators and IS for measurement were prepared in bovine serum with the same proportion of antioxidant solution as used for the human plasma samples to be analysed. Quantification was performed after precipitation and online solid phase extraction using reversed phase HPLC with tandem mass spectrometric detection.

\section{Results and discussion}

Temperature increase and particle size distribution in relation to the processing time as shown in Figure 2. According to broken cell wall theory the intrinsic heat energy increases the temperature of the cell and the wall will rupture thus releasing the phenolic compounds in the aqueous phase..$^{15}$ The patented hydrodynamic equipment reduced the particle size uniformly in to nano particles with in time period of 60 minutes. The particle size was measured, and temperature was monitored at time interval of each five minutes as shown in Table 1. This method has an advantage of uniform particle distribution which enables the maximum extraction efficiency. Achieved the good yield of $3-4 \%$ compared to the $1-2 \%$ yield of other conventional methods.

\section{Plasma concentration of total catechins}

Bioavailability parameters, including the area under the curve (AUC), the maximum observed concentration $\left(\mathrm{C}_{\mathrm{max}}\right)$ and time of maximum concentration $\left(\mathrm{T}_{\max }\right)$ for the three study formulations was calculated. Participants withdrawing prior to completing all three periods of this cross over study was excluded from the repeated measures analysis of variance. Probability values less than 0.05 was considered to be statistically significant. The statistical analysis was performed using industry recognized statistical software's.

The extract produced by Nanoemulsion method was found to have a significantly higher degree of bioavailability, i.e. the level of catechin absorption and retention in the blood stream, than other green tea extracts.

Table I Plasma concentration ( $\mathrm{ng} / \mathrm{ml}$ of plasma) of total catechins of reference green tea extract, hydyne processed extract and nano formulation

\begin{tabular}{|c|c|c|c|c|}
\hline \multirow{2}{*}{$\begin{array}{l}\text { Catechins } \\
\text { fraction }\end{array}$} & \multirow{2}{*}{$\begin{array}{l}\text { Time after } \\
\text { administration } \\
\text { (in minutes) }\end{array}$} & \multicolumn{3}{|c|}{ Plasma concentration ( $\mathrm{ng} / \mathrm{ml}$ of plasma) } \\
\hline & & Reference extract (sI) & Hydyne processed extract (s2) & Nano emulsion formulation (s3) \\
\hline \multirow[t]{8}{*}{ Total catechins } & 10 & 15 & 89 & 750 \\
\hline & 25 & 50 & 480 & 980 \\
\hline & 50 & 180 & 520 & 1250 \\
\hline & 100 & 100 & 980 & 1350 \\
\hline & 200 & 100 & 620 & 1580 \\
\hline & 400 & 200 & 480 & 1100 \\
\hline & 800 & 40 & 320 & 1000 \\
\hline & 1400 & 35 & 180 & 600 \\
\hline
\end{tabular}

The Hydyne produced green tea extract and the Nanoemulsion formulation, showed a greater overall bioavailability (Figure 2). The extract has shown that catechin concentrations peak 1 to 2 hours after ingestion and gradually reduce to undetectable levels after 24 hours as shown (Tables 1) below. An extract that allows the body to experience more of the health benefits of green tea is expected to have significant advantages over traditional green tea products where most of the catechins are lost through metabolization before they enter the bloodstream.

\section{Plasma concentration of total catechins}

Table ....and graph... shows the plasma concentration of total catechins of commercially available green tea extract (s1) (reference extract), Hydyne processed green tea extract (s2) and nano formulation of the Hydyne produced green tea extract (s3). Ten minutes after the oral administration, the plasma concentration of s1 was $15 \mathrm{ng} / \mathrm{ml}$, where as it was $89 \mathrm{ng} / \mathrm{ml}$ and $750 \mathrm{ng} / \mathrm{ml}$ in s2 and s3 respectively. This is very significant absorption rate in case of s 2 and s 3 . The reference extract (s1) showed an irregular plasma concentration at 10 ,
$25,50,100,200,400,800$ and 1400 minutes after oral administration. A peak concentration of $200 \mathrm{ng} / \mathrm{ml}$ of plasma was observed at 400 minutes after administration and $180 \mathrm{ng} / \mathrm{ml}$ of plasma concentration was observed at 50 minutes after administration. The plasma concentration was declined to $35 \mathrm{ng} / \mathrm{ml}$ of plasma at 1400 minutes after oral administration.

In contrast to s1, the samples s2 and s 3 showed a gradual increase in the plasma concentration with respect to time after oral administration. In s 2, it was $89.00 \mathrm{ng} / \mathrm{ml}$ of plasma at 10 minutes after oral administration, which gradually increased to $980.00 \mathrm{ng} / \mathrm{ml}$ plasma at 100 minutes after oral administration, which then gradually decreased to $620.00,480.00,320.00$ and $180.00 \mathrm{ng} / \mathrm{ml}$ plasma at $200,400,800$ and 1400 minutes after oral administration.

A more similar tread was observed in $\mathrm{s} 3$; but the increase of plasma concentration in s 3 was 10 fold higher than s2. At 10 minutes after oral administration, the plasma concentration was $750 \mathrm{ng} / \mathrm{ml}$ of plasma. This gradually increased to $980.00,1250.00,1350.00$ and $1580.00 \mathrm{ng} /$ $\mathrm{ml}$ of plasma at 25, 50, 100 and 200 minutes after oral administration, 
respectively. There observed a declining trend from 400, 800 and 1400 minutes after oral administration (1100.00, 1000.00 and 600.00 $\mathrm{ng} / \mathrm{ml}$ of plasma).

\section{Acknowledgments}

None.

\section{Conflicts of interest}

The author declares that there is no conflict of interest.

\section{Funding}

None.

\section{References}

1. Willcox M, Burford G, Bodeker G. An overview of ethnobotanical studies on plants used for the treatment of malaria. In Traditional Medicinal Plants and Malaria. Boca Raton: CRC Press; Willcox M, Bodeker G, Rasoanaivo P, editors. 2004:187-197.

2. Batista R, Silva AdJ Jr, de Oliveira AB. Plant-derived antimalarial agents: new leads and efficient phytomedicines. Part II. Non-alkaloidal natural products. Molecules. 2009;14:3037-3072.

3. Kaur K, Jain M, Kaur T, et al. Antimalarials from nature. Bioorganic \& Medicinal Chemistry. 2009, 17:3229-3256.

4. Oliveira AB, Dolabela MF, Braga FC, et al. Plant-derived antimalarial agents: new leads and efficient phythomedicines. Part I. Alkaloids. Anais da Academia Brasileira de Ciencias. 2009; 81:715-740.

5. Sannella Anna, Messori, Luigi, Casini Angela, et al. Antimalarial properties of green tea. Biochemical and biophysical research communications. 2007;353:177-181
6. Hong J, Lambert JD, Lee SH, et al. Involvement of multidrug resistanceassociated proteins in regulating cellular levels of (-)-epigallocatechin-3gallate and its methyl metabolites. Biochem Biophys Res Commun. 2003;310:222-227.

7. Tasdemir D, Lack G, Brun R, et al. Inhibition of Plasmodium falciparumfatty acid biosynthesis: evaluation of FabG, FabZ, and FabI as drug targets for flavonoids. J MedChem. 2006; 49:3345-3353.

8. Lante A, Friso D. Oxidative stability and rheological properties of nanoemulsions with ultrasonic extracted green tea infusion. Food ResearchInternational. 2013;54:269-276.

9. Lambert Joshua, Hong JG, Kim DH, et al. Piperine enhances the bioavailability of the tea polyphenol (-)-epigallocatechin-3-gallate in mice. Journal of Nutrition. 2004;134:1948-1952.

10. Komaiko J, McClements DJ. Low-energy formation of edible nanoemulsions by spontaneous emulsification: Factors influencing particle size. Journal of Food Engineering. 2015;146:122-128.

11. Huang Q, Yu H, Ru Q. Bioavailability and delivery of nutraceuticals using nanotechnology. Journal of Food Science. 2010;75:R50-R57.

12. Jaiswal M, Dudhe R, Sharma PK. Nanoemulsion: An advanced mode of drug delivery system. 3 Biotech. 2015;5:123-127.

13. Vardin Hasan, Ikinci Ali, Dalgıç Ali, et al. Drying kinetics and thermal degradation of phenolic compounds and anthocyanins in pomegranate arils dried under vacuum conditions. International Journal of Food Science \& Technology. 2014;49.

14. McDonald Suzy, Prenzler Paul, Antolovich Michael, et al. Phenolic content and anti-oxidant activity of olive extract. Food Chemistry. 2001;73:73-84.

15. https://bioeng.berkeley.edu/about-us/what-is-bioengineering 